\title{
Pre-SMTP, a key precursor for the biosynthesis of the SMTP plasminogen modulators
}

\author{
Yuuichi Nishimura ${ }^{1}$, Eriko Suzuki ${ }^{1}$, Keiko Hasegawa ${ }^{2}$, Naoko Nishimura ${ }^{2}$, Yoshikazu Kitano ${ }^{1}$ \\ and Keiji Hasumi ${ }^{1,2}$
}

The Journal of Antibiotics (2012) 65, 483-485; doi:10.1038/ja.2012.47; published online 20 June 2012

Keywords: biosynthesis; phthalimidine; plasminogen modulator; SMTP

SMTPs are triprenyl phenol metabolites obtained from the fungus Stachybotrys microspora. ${ }^{1}$ SMTP enhances the activation of plasminogen by modulating its conformation. ${ }^{1-4}$ SMTP-7, one of the most potent congeners, is effective in enhancing blood clot clearance and treating thrombotic stroke. ${ }^{4-8}$ The SMTP molecule consists of a tricyclic $\gamma$-lactam moiety, a geranylmethyl group and an $N$-linked side chain. Our previous studies identified 30 SMTP congeners, which differ in the $N$-linked side chain. ${ }^{9-16}$ Plasminogen modulator activities of the congeners differ depending on the $N$-linked side-chain structure. ${ }^{15,16}$ These congeners can be produced by a precursor amine-fed culture. ${ }^{17}$ In this method, some metabolites existing in the culture are consumed following the amine feeding, with a concomitant formation of an SMTP congener, which has the fed amine as the $N$-linked side chain. Thus, the presence of a key precursor for the biosynthesis of SMTPs is suggested. In this study, we isolated a compound, designated pre-SMTP (1), which directly affords SMTP congeners by reacting nonenzymatically with amines.

In the precursor amine-fed culture, S. microspora is grown in medium containing limited amounts of amine compounds before amine feeding. During this "starvation phase", significant amounts of possible precursors are accumulated. We purified two of the candidates, compounds 1 and 2 (Figure 1a), by solvent extraction<smiles>CC(C)=CCCC(C)=CCC[C@]1(C)Oc2c(cc(O)c3c2CNC3=O)CC1O</smiles><smiles>CC(C)=CCCC(C)=CCC[C@]1(C)Oc2c(C=O)c(C=O)cc(O)c2CC1O</smiles>

Pre-SMTP (1)<smiles>[Z10]C=[Z10]#CCCc1c(O)cc(C)c(C=O)c1O</smiles>

b

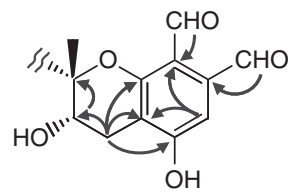<smiles>CC(C)=CCC/C(C)=C/CC[C@]1(C)Oc2c(cc(O)c3c2CN([C@@H](CCC(N)=O)C(=O)O)C3=O)C[C@H]1O</smiles>

SMTP-54 (3)<smiles>CC(C)=CCCC(C)=CCC[C@]1(C)Oc2c(cc(O)c3c2CN([C@@H](CCC(=O)O)C(C)(C)C)C3=O)C[C@H]1O</smiles>

SMTP-55 (4)

Figure 1 Structures of SMTP-0, pre-SMTP (1), LL-Z1272 $\beta$ (2), SMTP-54 (3) and SMTP-55 (4). (a) Structures of SMTP-0, pre-SMTP (1) and LL-Z1272 $\beta$ (2). (b) Key ${ }^{1} \mathrm{H}-{ }^{13} \mathrm{C}$ correlations observed in the HMBC spectrum of 1 . (c) Structures of SMTP-54 (3) and SMTP-55 (4), which are derived from 1 through nonenzymatic reaction with L-glutamine and L-glutamic acid, respectively.

${ }^{1}$ Department of Applied Biological Science, Tokyo Noko University, Tokyo, Japan and 2Division of Research and Development, TMS Co. Ltd., Tokyo, Japan Correspondence: Dr K Hasumi, Department of Applied Biological Science, Tokyo Noko University, 3-5-8 Saiwaicho, Fuchu, Tokyo 183-8509, Japan. E-mail: hasumi@cc.tuat.ac.jp

Received 31 January 2012; revised 25 April 2012; accepted 17 May 2012; published online 20 June 2012 
and reversed-phase HPLC (see Supplementary Methods and Results). The physicochemical properties (Supplementary Table S1) and the NMR spectra of 1 (Supplementary Table S2 and Supplementary Figure S1) suggested that $\mathbf{1}$ is a new compound. The physicochemical properties of $\mathbf{2}$ (Supplementary Methods and Results) were consistent with those of LL-Z1272 $\beta$, which was isolated from Fusarium sp. as an antiprotozoan. ${ }^{18}$ The presence of a geranylmethyl group and a chroman skeleton in the structure of $\mathbf{1}$, which were identical to the partial structure of the SMTP family compounds, was confirmed by the NMR spectral data, including hetero-nuclear multiple-quantum coherence and HMBC spectroscopies. The NMR data suggested that 1 did not have the lactam structure, as the carbonyl signal at position 2 $\left(\delta_{\mathrm{c}} 170.24\right)$ and the methylene signals at position $13\left(\delta_{\mathrm{c}} 41.95 ; \delta_{\mathrm{H}} 4.09\right.$ and 4.05) present in SMTP-0 were missing. Instead, the low-field signals at $\delta_{\mathrm{c}} 193.06$ and 190.06 , as well as $\delta_{\mathrm{H}} 10.46(1 \mathrm{H}, \mathrm{s})$ and 10.37 $(1 \mathrm{H}, \mathrm{s})$, suggested the presence of two aldehyde groups. An $o$-dialdehyde structure and the relative position of the two low-field oxygen-bearing olefinic carbons $\left(\delta_{c} 161.96\right.$ and 159.17) and the olefinic methine $\left(\delta_{\mathrm{c}} 106.66 ; \delta_{\mathrm{H}} 6.85\right)$ were confirmed by the HMBC spectrum (Figure 1b). On the basis of these observations, the structure of 1 was elucidated as shown in Figure 1. The stereochemistries at positions 8 and 9 shown in Figure 1 were based on the fact that 1 afforded SMTP-0, SMTP-4 and SMTP-6 on reaction with ammonium chloride, L-phenylalanine and L-tryptophan, respectively (see below). Thus, we designate compound 1 pre-SMTP.

The structural features of $\mathbf{1}$ and $\mathbf{2}$, and the fact that these compounds are rapidly consumed following the amine feeding, suggest that these compounds are precursors of SMTPs. Compound 2 has an uncyclized farnesyl side chain, and hence is assumed to be a precursor of pre-SMTP, which has a geranylmethyl side chain attached to a chroman structure. In addition, 2 lacks $8-\mathrm{OH}$ and has a methyl group instead of an aldehyde group in 1 . Thus, there may be at least 3 steps, a cyclization, a hydroxylation (position 8 ) and an oxidation (position 2), in the conversion of $\mathbf{2}$ to $\mathbf{1}$.

Although SMTP-7 enhanced the plasminogen activation catalyzed by urokinase-type plasminogen activator activities of compounds 1 and $\mathbf{2}$ in promoting plasminogen activation were minimal (Supplementary Figure S2).

Pre-SMTP (1) has an $o$-phthalaldehyde partial structure, which can react with an amine compound to afford a phthalimidine structure such as that present in SMTPs. The formation of phthalimidines via a 1:1 condensation between $o$-phthalaldehyde and several kinds of amines has been reported to proceed under mild conditions. ${ }^{19,20}$ Therefore, we tested $\mathbf{1}$ for reaction with some amines. Although $\mathbf{1}$ was stable in the absence of amine in buffer ( $20 \mathrm{~mm}$ potassium phosphate, $\mathrm{pH} 7.4$ ) or $50 \%$ aqueous acetone in the presence or absence of acetic acid $(1 \%, v / v)$ (Figure $2 a)$, the addition of ammonium acetate resulted in a decrease in pre-SMTP and a concomitant formation of SMTP-0 (Figure 2b) under these three conditions. Similarly, the reaction between 1 and L-phenylalanine and L-tryptophan afforded SMTP-4 and SMTP-6, respectively (Figures $2 \mathrm{c}$ and $\mathrm{d}$ ). These results suggest that $\mathbf{1}$ is a direct precursor of SMTPs.

The formation of SMTPs through nonenzymatic reaction between 1 and an amine enables the synthesis of a variety of congeners with different $\mathrm{N}$-linked side-chain structures. The microbial production of SMTP congeners through the precursor amine-feeding method cannot be adapted for certain amines, possibly because of the problems with cellular uptake. For example, congeners with L-glutamine and L-glutamic acid as the $N$-linked side chain have not been produced through the precursor amine-feeding method. Nevertheless, the nonenzymatic reaction between 1 and these amines
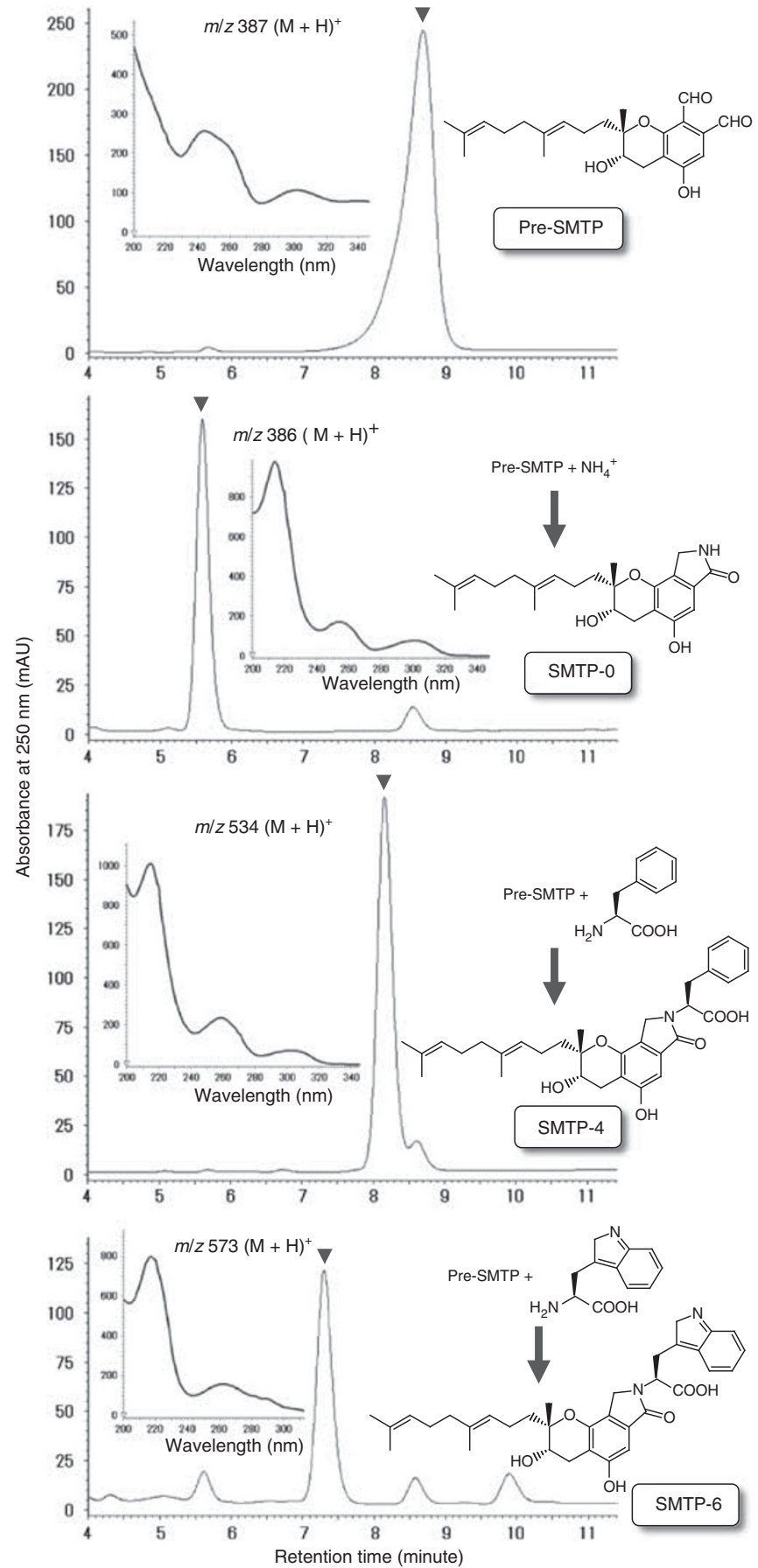

Figure 2 Conversion of pre-SMTP (1) to SMTPs. Pre-SMTP $\left(100 \mu \mathrm{g} \mathrm{ml}^{-1}\right.$ in acetone) was incubated with 1 volume of water (a), ammonium acetate (b), L-phenylalanine (c) or L-tryptophan (d) in the presence of $1.5 \%(\mathrm{v} / \mathrm{v})$ acetic acid, and the products were analyzed by HPLC. The arrowhead indicates the peak at which UV spectrum (shown in the inset) and mass spectrum were taken. The elution positions, UV spectra and mass spectra of the indicated peaks in panels $(\mathbf{a}-\mathbf{d})$ were consistent with those of authentic pre-SMTP, SMTP-0, SMTP-4 and SMTP-6, respectively. Essentially the same results were obtained with the incubations of pre-SMTP and amines in the absence of acetic acid or the incubation in buffer, where acetone concentration was $1 \%(\mathrm{v} / \mathrm{v})$.

afforded congeners with an expected side chain, SMTP-54 and SMTP 55, respectively (Figure 1c; Supplementary Methods and Results; Supplementary Table S3; Supplementary Figures S3 and S4). 
1 Hasumi, K., Yamamichi, S. \& Harada, T. Small molecule modulators of the zymogen activation in the fibrinolytic and coagulation systems. FEBS J. 277, 3675-3687 (2010).

2 Takayasu, R., Hasumi, K., Shinohara, C. \& Endo, A. Enhancement of fibrin binding and activation of plasminogen by staplabin through induction of a conformational change in plasminogen. FEBS Lett. 418, 58-62 (1997).

3 Ohyama, S., Harada, T., Chikanishi, T., Miura, Y. \& Hasumi, K. Nonlysine-analog plasminogen modulators promote autocatalytic generation of plasmin(ogen) fragments with angiostatin-like activity. Eur. J. Biochem. 271, 809-820 (2004).

$4 \mathrm{Hu}$, W., Narasaki, R., Nishimura, N. \& Hasumi, K. SMTP (Stachybotrys microspora triprenyl phenol) enhances clot clearance in a pulmonary embolism model in rats. Thromb. J. 10, 2 (2012).

5 Hashimoto, T., Shibata, K., Nobe, K., Hasumi, K. \& Honda, K. A novel embolic model of cerebral infarction and evaluation of SMTP-7, a novel fungal triprenyl phenol metabolite. J. Pharmacol. Sci. 114, 41-49 (2010).

6 Shibata, K., Hashimoto, T., Nobe, K., Hasumi, K. \& Honda, K. A novel finding of a lowmolecular-weight compound, SMTP-7, having thrombolytic and anti-inflammatory effects in cerebral infarction of mice. N.-S. Arch. Pharmacol. 382, 245-253 (2010).

7 Miyazaki, T. et al. Distinct effects of tissue-type plasminogen activator and SMTP-7 on cerebrovascular inflammation following thrombolytic reperfusion. Stroke 42, 1097-1104 (2011).

8 Shibata, K., Hashimoto, T., Nobe, K., Hasumi, K. \& Honda, K. Neuroprotective mechanisms of SMTP-7 in cerebral infarction model in mice. N.-S. Arch. Pharmacol. 384, 103-108 (2011).

9 Shinohara, C., Hasumi, K., Hatsumi, W. \& Endo, A. Staplabin, a novel fungal triprenyl phenol which stimulates the binding of plasminogen to fibrin and U937 cells. J. Antibiot. 49, 961-966 (1996).

10 Kohyama, T., Hasumi, K., Hamanaka, A. \& Endo, A. SMTP-1 and -2, novel analogs of staplabin produced by Stachybotrys microspora IF030018. J. Antibiot. 50, 172-174 (1997).
11 Hasumi, K. et al. Isolation of SMTP-3, $-4,-5$ and -6 , novel analogs of staplabin, and their effects on plasminogen activation and fibrinolysis. J. Antibiot. 51, 1059-1068 (1998).

$12 \mathrm{Hu}$, W., Ohyama, S. \& Hasumi, K. Activation of fibrinolysis by SMTP-7 and -8, novel staplabin analogs with a pseudosymmetric structure. J. Antibiot. 53, 241-247 (2000).

$13 \mathrm{Hu}$, W., Kitano, Y. \& Hasumi, K. SMTP-4D, -5D, -6D, -7D and -8D, a new series of the non-lysine-analog plasminogen modulators with a D-amino acid moiety. J. Antibiot. 56, 832-837 (2003).

14 Hasumi, K., Hasegawa, K. \& Kitano, Y. Isolation and absolute configuration of SMTP-0, a simplest congener of the SMTP family nonlysine-analog plasminogen modulators. J. Antibiot. 60, 463-468 (2007).

15 Hasegawa, K. et al. Structure-activity relationships of fourteen new congeners of the SMTP plasminogen modulator. J. Antibiot. 63, 589-593 (2010).

16 Koide, H., Narasaki, R., Hasegawa, K., Nishimura, N. \& Hasumi, K. A new series of the SMTP plasminogen modulator with a phenylglycine-based side-chain. J. Antibiot. 65, 91-93.

$17 \mathrm{Hu}$, W., Ohyama, S., Narasaki, R. \& Hasumi, K. Selective production of staplabin and SMTPs in cultures of Stachybotrys microspora fed with precursor amines. J. Antibiot. 54, 962-966 (2001).

18 Ellestad, G. A., Evans, Jr. R. H. \& Kunstmann, M. P. Terpenoid metabolites from an unidentified Fusarium species. Tetrahedron 25, 1323-1334 (1969).

19 Alajarín, M., Sánchez-Andrada, P., López-Leonardo, C. \& Álvarez, Á. On the mechanism of phthalimidine formation via o-phthalaldehyde monoimines. New $[1,5]-\mathrm{H}$ sigmatropic rearrangements in molecules with 5-aza-2,4-pentadienal skeleton. J. Org. Chem. 70, 7617-7623 (2005).

20 Gyimesi-Forrás, K., Leitner, A., Akasaka, K. \& Lindner, W. Comparative study on the use of ortho-phthalaldehyde, naphthalene-2,3-dicarboxaldehyde and anthracene-2,3dicarboxaldehyde reagents for $\alpha$-amino acids followed by the enantiomer separation of the formed isoindolin-1-one derivatives using quinine-type chiral stationary phases. J. Chromatogr. A. 1083, 80-88 (2005).

Supplementary Information accompanies the paper on The Journal of Antibiotics website (http://www.nature.com/ja) 